\title{
Comparison of Histochemical Staining Methods and Correlation with Transient Elastography in Acute Hepatitis
}

\author{
Daniela Cabibi $^{a}$ Vincenza Calvaruso $^{b}$ Letizia Giuffrida $^{a}$ Sabrina Ingrao ${ }^{a}$ \\ Laura Balsamo $^{a}$ Antonino Giulio Giannone ${ }^{a}$ Salvatore Petta ${ }^{b}$ Vito Di Marco ${ }^{b}$ \\ ${ }^{a}$ Human Pathology Section, Department of Science for Promotion of Health and Mother and Child Care, and \\ ${ }^{b}$ Gastroenterology and Hepatology Unit, Biomedical Department of Internal Medicine and Medical Specialties, \\ University of Palermo, Palermo, Italy
}

\section{Key Words}

Acute hepatitis · Digital image analysis · Elastic fibrosis . Liver stiffness measurements · Orcein staining · Staining methods · Transient elastography

\begin{abstract}
Objective: To compare Masson's trichrome (MT), Sirius red (SR) and orcein staining in acute hepatitis (AH) and to correlate them with transient elastography (TE), a noninvasive method to assess hepatic fibrosis. Methods: We evaluated liver stiffness by TE in a cohort of 34 consecutive patients and assessed MT-, SR- and orcein-stained biopsies using the METAVIR scoring system and digital image analysis (DIA). $\boldsymbol{R e}$ sults: MT and SR both showed severe fibrosis (stage III-IV, $\mathrm{DIA}=12.7 \%$ ). Orcein showed absent or mild fibrosis (stage $0-I I, \mathrm{DIA}=4.4 \% ; \mathrm{p}<0.05)$. In 29/34 cases (85\%), stiffness values were $>12.5 \mathrm{kPa}$, in keeping with SR/MT but not with orcein results. Conclusions: Even though in AH true elastic fibrosis is typically absent or mild, TE shows elevated stiffness values, in keeping with SR/MT evaluations. If not properly evaluated in the clinical context, these results would lead to an overestimation of fibrosis. Orcein is the only staining able
\end{abstract}

to evidence the absence of true elastic fibrosis, which is a typical feature of $\mathrm{AH}$. This is the first study comparing different staining procedures performed on $\mathrm{AH}$ biopsies by DIA versus TE.

(C) 2015 S. Karger AG, Basel

\section{Introduction}

The utility of liver biopsy in diagnosing acute liver damage is still subject to controversy. Acute hepatitis (AH) lacks significant elastic fibrosis by definition [1] and pathologists usually perform more than one staining to evaluate liver fibrosis, such as silver impregnation for reticulin fibers, chromotrope aniline blue for collagen, and Masson's trichrome (MT), picro-Sirius red (SR), Victoria blue or orcein stain for elastic fibers.

The results of these staining methods are not always congruent because they highlight different connective components, which increase during the progression of AH. SR and MT staining highlight the collagenous component of liver fibrosis, whereas, according to Scheuer and Lefkowtich [1], Victoria blue or orcein staining are

\section{5-2008/15/0821-0048\$39.50/0}


important to distinguish between recent and old fibrosis because they highlight elastic fibers, which may be considered the product of true active formation of new septa. Elastic fibers are usually absent in normal portal tracts. Nevertheless, proper orcein staining is sometimes difficult and depends on the technician's skills. Other techniques have been introduced with the aim to best quantify liver fibrosis, such as computer-assisted digital image analysis (DIA) of liver collagen, in which the quantitative assessment of liver fibrosis is usually performed on SRstained sections, with SR being the preferred histochemical method when quantifying liver fibrosis $[2,3]$. As Standish et al. [3] report 'Some groups have used reticulin or trichrome stains, and others have suggested the use of immunohistochemistry but, due to variability in staining and difficulty in accurate thresholding, these techniques can suffer from 'poor reproducibility'. Moreover, previous studies showed that the amount of liver collagen obtained with DIA is significantly correlated with clinical outcome in chronic hepatitis $[4,5]$.

Finally, transient elastography (TE), a recent noninvasive method to assess hepatic fibrosis by FibroScan (Echosens) [6], has found wide acceptance in the clinical management of liver diseases and, in some studies, TE values in AH were strongly elevated, achieving values usually in the diagnostic range of liver cirrhosis [79].

However, no studies have been performed to assess if the above-mentioned staining methods, which were used to evaluate liver fibrosis semiquantitatively and quantitatively, correlate with TE results in $\mathrm{AH}$.

The aim of this study is to compare MT-, SR- and orcein-stained slides of $\mathrm{AH}$ cases using semiquantitative and quantitative methods. Furthermore, we would like to verify if liver stiffness assessed by TE correlates with the results obtained using the different histological staining methods.

\section{Materials and Methods}

This prospective study assessed consecutive samples of liver biopsies obtained from patients with a clinical diagnosis of $\mathrm{AH}$ made at the Pathology Department, University Hospital of Palermo, collected between January 2009 and December 2012.

One hundred and seven cases of AH [defined by increased alanine aminotransferase levels (ALT; $>10 \times$ the upper limit of normal) for a period less than 6 months and without a previous history of liver disease] have been observed during the enrollment period, and 34 of the 107 patients underwent liver biopsy in order to better define the etiology of liver damage with the help of histological features.

Differences in Staining Results in $\mathrm{AH}$
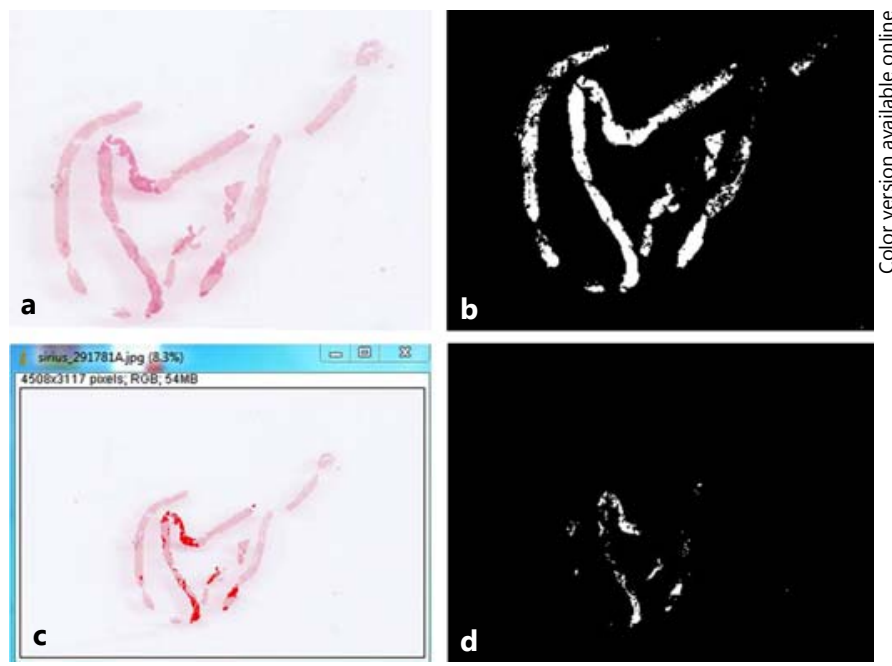

Fig. 1. a Digitized image of the entire scanned section. SR. b Selection of the area of the entire scanned section. c, $\mathbf{d}$ Selection of the SR-stained fibrous areas.

Indeed, in common clinical practice as well as for ethical considerations, no patient was subjected to liver biopsy when the diagnosis and the pathogenesis of the disease was clinically well defined. Due to the small number of AH cases biopsied in our casuistry, we included and analyzed $\mathrm{AH}$ cases of different pathogenesis: 17 drugrelated cases (nonsteroidal anti-inflammatory drugs in 13 cases and amoxicillin in 4 cases); 13 autoimmune hepatitis cases; 2 acute HCV-related hepatitis cases and 2 cases of unknown etiology which, for convenience, have been merged into a single group.

The slides were stained with hematoxylin-eosin, Shikata's orcein, MT and SR. Previously reported protocols were applied for these staining methods $[1,10]$.

SR staining is obtained by dissolving $0.5 \mathrm{~g}$ SR in $500 \mathrm{ml}$ of saturated aqueous solution of picric acid. It is specific for many types of collagen (types I, II, III, IV and V). We did not counterstain the nuclei with hematoxylin because the addition of another color could render DIA difficult. In this way, SR is useful to quantify total collagen in liver biopsies due to the dual-color staining obtained, with collagen fibers staining red and parenchyma light pink.

We used the METAVIR scoring system $[11,12]$ (originally performed in chronic hepatitis $\mathrm{C}$ ) for the semiquantitative assessment of fibrosis in AH. Shikata's orcein-, SR- and MT-stained slides were separately evaluated by a liver pathologist (D.C.) blinded to the TE results. We preferred orcein to Victoria blue staining for elastic fibers, because orcein staining, like SR, provides better staining for DIA compared with MT $[2,3]$.

DIA was performed in all $34 \mathrm{AH}$ cases using the Zeiss Axio Vision Rel 4.8 image analysis software on digitized images of Shikata's orcein- and SR-stained sections by V.C., who was blinded to clinical, laboratory and histological information. Therefore, the entire slides were scanned through a histological slide scanner.

Quantitative analysis involves the following steps (fig. 1):

- Acquisition of the entire section as a scanned image.

- Image processing and quantitative measurement of the stained fibrous areas (using AxioVision software).

- Percentage ratio of the fibrotic area to the entire sample. 


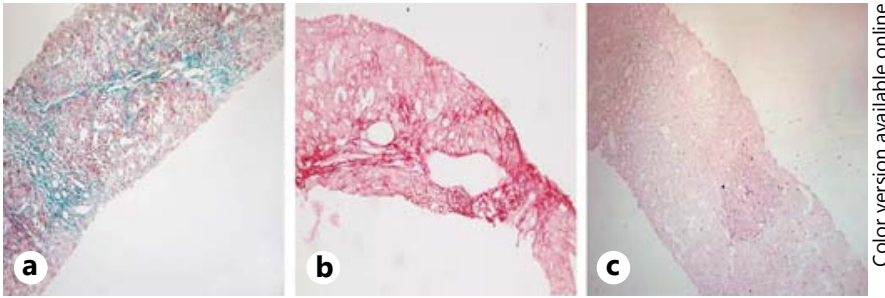

Fig. 2. AH: MT (a) and SR (b) showing bridging fibrosis, suggesting stage III-IV fibrosis. c Absence of fibrosis with Shikata's orcein staining. a-c Original magnification $\times 200$.

Statistical analysis of the results was performed using Student's t test with SPSS 15.0. Continuous variables are expressed as means \pm $\mathrm{SD}$ and categorical variables as absolute and relative frequencies. The differences between continuous data were analyzed by t test, and corrected $\chi^{2}$ analysis was used for dichotomous or categorical variables. The results were considered statistically significant at $\mathrm{p}<0.05$.

Liver stiffness was assessed in all cases at the time of biopsy with a FibroScan (Echosens, Paris). In AH, where liver stiffness values are usually high and similar to the results found in cirrhotic patients, we used a cutoff value of $12.5 \mathrm{kPa}$ [13] for comparisons with histological findings.

\section{Results}

Table 1 lists clinical and histological features of the patients included in the study. In all 34 cases, necroinflammatory activity, which was assessed on HE slides, showed a severe grade with spotty and bridging necrosis. MT and SR staining showed the same results with respect to liver fibrosis in all $\mathrm{AH}$ cases (percentage of concordance $100 \%)$. Hence, the results of the two staining methods, MT and SR, are reported together.

A clear discrepancy was evident between MT/SR staining and Shikata's orcein staining, with the former always showing bridging fibrosis, suggesting stage III-IV, and the latter suggesting the absence or scanty presence of fibrosis (stage 0-II; fig. 2).

These semiquantitative observations were confirmed by DIA, in which the average percentages of SR-stained areas were significantly greater than the orcein-stained areas: SR $12.7 \%( \pm 5.6 \%)$ and Shikata's orcein $4.4 \%( \pm 7.2 \%$; $\mathrm{p}<0.035)$. This statistically significant difference persists after dividing patients according to $\mathrm{AH}$ etiology (table 2).

\section{Correlation with TE}

In $29 / 34 \mathrm{AH}$ cases, TE values were $\geq 12.5 \mathrm{kPa}$. This was in keeping with the stage III-IV suggested by the semiquantitative assessment of liver fibrosis on MT/SR but

Table 1. Clinical and histological features of AH patients $(n=34)$

Age, years

Male gender, $n$

ALT, U/l

Platelets, $\times 10^{9} / 1$

r-Glutamyl transferase, U/l

$\mathrm{TE}, \mathrm{kPa}$

Grade of inflammation

$\begin{array}{lc}1 & 0 \\ 2 & 0 \\ 3 & 11(32.4 \%) \\ 4 & 23(67.6 \%) \\ \text { Collagen proportionate area, } \% & \\ \text { SR } & 12.7 \pm 5.6 \\ \text { Orcein } & 4.4 \pm 7.2\end{array}$

Orcein

Means \pm SD and numbers of patients (\%) are shown.

Table 2. Comparison of DIA results of SR/MT- and orcein-stained slides in $\mathrm{AH}$ (means $\pm \mathrm{SD})$

\begin{tabular}{llll}
\hline & $\begin{array}{l}\text { SR/MT, } \\
\%\end{array}$ & $\begin{array}{l}\text { Orcein, } \\
\%\end{array}$ & $\begin{array}{l}\mathrm{p} \\
\text { value }\end{array}$ \\
\hline AH $(\mathrm{n}=34)$ & $12.7 \pm 5.6$ & $4.4 \pm 7.2$ & 0.029 \\
Drug-induced AH $(\mathrm{n}=17)$ & $13.7 \pm 5.1$ & $4.6 \pm 6.5$ & 0.036 \\
Autoimmune AH $(\mathrm{n}=13)$ & $11.8 \pm 4.8$ & $4.2 \pm 7.7$ & 0.043 \\
Other AH $(\mathrm{n}=4)$ & $12.8 \pm 5.0$ & $4.1 \pm 8.7$ & 0.031 \\
\hline
\end{tabular}

Table 3. FibroScan values and histological fibrosis evaluation with SR/MT and orcein staining in $34 \mathrm{AH}$ patients

\begin{tabular}{llll}
\hline & Stage & FibroScan & \\
\cline { 3 - 4 } & & $\geq 12.5 \mathrm{kPa}$ & $<12.5 \mathrm{kPa}$ \\
\hline AH & 29 & 5 \\
SR/MT & I-II & 0 & 0 \\
\multirow{2}{*}{ Orcein } & III-IV & 29 & 5 \\
& I-II & 29 & 5 \\
& III-IV & 0 & 0 \\
\hline
\end{tabular}

not with orcein staining, which indicated the absence of severe fibrosis in all cases ( 21 cases were evaluated as stage $0-$ I, 11 cases as stage II and there was no case of stage IIIIV; table 3).

Using linear regression analysis, we have not found a significant correlation between TE and ALT values in our AH patients $\left(\mathrm{r}^{2}=0.007 ; \mathrm{p}=0.796\right)$.
Cabibi/Calvaruso/Giuffrida/Ingrao/ Balsamo/Giannone/Petta/Di Marco 


\section{Discussion}

Routine fibrosis assessment is usually carried out based on MT or reticulin staining [3]. However, the issue of elastic fiber staining has been stressed previously since, in case of parenchymal collapse simulating septa, collagen stains could lead to a misdiagnosis of cirrhosis $[1,14]$. In our study on $\mathrm{AH}, \mathrm{SR} / \mathrm{MT}$ showed a high level of discordance with orcein staining. Indeed, SR/MT indicated advanced fibrosis (stage III-IV), which was not supported by the paired orcein-stained slides, in which fibrosis was absent or low/moderate (stage $0-\mathrm{II})$. These findings were confirmed by quantitative analysis showing on average $12.7 \%$ SR-positive areas versus $4.4 \%$ orcein-positive areas $(\mathrm{p}<0.05)$.

Moreover, in $\mathrm{AH}$, the advanced fibrosis observed by $\mathrm{SR} / \mathrm{MT}$ is associated with high stiffness values $(12.5 \mathrm{kPa})$, whereas orcein staining correctly shows absent or mild fibrosis. Previous studies reported that in $\mathrm{AH}$ or chronic hepatitis of viral origin with severe necroinflammation, liver stiffness values are greatly increased and correlate with high values of aminotransferases [7-9].

Arena et al. [7] hypothesized that liver stiffness measurements (LSM) were correlated with aminotransferase levels at the onset of acute viral hepatitis due to the presence of tissue inflammation and edema, which at this time are likely to be maximal. Therefore, they highlighted 'the contribution of nonfibrotic modifications to LSM, supported by a progressive normalization of stiffness values in parallel with the decrease of aminotransferase levels'.

Noteworthy, for ethical considerations, they did not perform liver biopsy in $\mathrm{AH}$ cases and in their study acute liver damage could only be assessed by peak increases and subsequent changes in serum biochemical markers.

To our knowledge, this is the first study performed on liver biopsies of $\mathrm{AH}$ cases including staining with different methods in order to highlight the different components of fibrosis (collagen and elastic fibers). Morphological evidence provided by these staining methods allowed us to better understand the relationship between high levels of transaminases and FibroScan values.

In fact, high aminotransferases values are an index of severe necroinflammatory activity, which, in acute liver disease, results in connective texture collapse and the formation of collagen passive septa due to hepatocyte necrosis. These features are evidenced by SR/MT staining (both semiquantitatively and quantitatively by DIA) and could affect TE values. In keeping with the literature, in our study, 29/34 patients showed high stiffness values $(\geq 12.5$
$\mathrm{kPa}$ ) and, by definition, all our cases showed transaminase levels $>10 \times$ the upper limit of normal. However, we have not found a correlation between TE and ALT values in our cohort of AH patients, probably because in patients with markedly elevated ALT value, once collagen passive septa are consistently formed, a further increase in biochemical markers was no longer proportional to increasing stiffness. By contrast, orcein-stained elastic fibers are the product of true new active septum formation and are present only in older fibrosis. Thus, to achieve a diagnosis of $\mathrm{AH}$, which lacks significant elastic fibrosis by definition, orcein seems to be superior to both TE and SR/MT, since TE and SR/MT could lead to a misdiagnosis of severe fibrosis.

In our opinion, the relationship between peak increases in aminotransferase levels and liver stiffness may not simply be explained by 'the inflammatory infiltration, hepatocyte swelling and tissue edema', as previously hypothesized [7, 8], but rather by the formation of SR/MTpositive collagen passive septa, which are potentially reversible.

In conclusion, our study highlights the importance of orcein staining in $\mathrm{AH}$. Indeed, if not properly evaluated in the clinical context, SR/MT staining could lead to a wrong diagnosis, resulting in an overestimation of the staging, which is erroneously supported by high values of liver stiffness measurements.

\section{Disclosure Statement}

No potential conflict of interest to report.

References

1 Scheuer PJ, Lefkowtich JH: Liver Biopsy Interpretation, ed 6. London, Saunders, 2000.

$\checkmark 2$ Puchtler H, Meloan SN, Waldrop FS: Are picro dye reactions for collagens quantitative? Chemical and histochemical considerations. Histochemistry 1988;88:243-256.

3 Standish RA, Cholongitas E, Dhillon A, Burroughs AK, Dhillon AP: An appraisal of the histopathological assessment of liver fibrosis. Gut 2006;55:569-578.

4 Calvaruso V, Burroughs AK, Standish R, et al: Computer-assisted image analysis of liver collagen: relationship to Ishak scoring and hepatic venous pressure gradient. Hepatology 2009;49:1236-1244.

5 Calvaruso V, Dhillon AP, Tsochatzis E, et al: Liver collagen proportionate area predicts decompensation in patients with recurrent hepatitis $\mathrm{C}$ virus cirrhosis after liver transplantation. J Gastroenterol Hepatol 2012;27:1227-1232. 
6 Ziol M, Handra-Luca A, Kettaneh A, et al: Noninvasive assessment of liver fibrosis by measurement of stiffness in patients with chronic hepatitis C. Hepatology 2005;41:4854.

7 Arena U, Vizzutti F, Corti G, et al: Acute viral hepatitis increases liver stiffness values measured by transient elastography. Hepatology 2008;47:380-384.

8 Coco B, Oliveri F, Maina AM, et al: Transient elastography: a new surrogate marker of liver fibrosis influenced by major changes of transaminases. J Viral Hepat 2007;14:360-369.
-9 Di Marco V, Calvaruso V, Iacò A, et al: Liver stiffness measurement by transient elastography predicts early recovery from acute hepatitis. Gut 2011;60:1023.

10 Mazzi V: Manuale di tecniche istologiche e istochimiche. Padova, Piccin, 1977.

11 Bedossa P, Bioulac-Sage P, Callard P, et al: Intraobserver and interobserver variations in liver biopsy interpretation in patients with chronic hepatitis C. Hepatology 1994;20:1520.
2 Bedossa P, Poynard T: An algorithm for the grading of activity in chronic hepatitis $\mathrm{C}$. The METAVIR Cooperative Study Group. Hepatology 1996;24:289-293.

13 Castéra L, Vergniol J, Foucher J, Le Bail B, Chanteloup E, Haaser M, et al: Prospective comparison of transient elastography, Fibrotest, APRI, and liver biopsy for the assessment of fibrosis in chronic hepatitis C. Gastroenterology $2005 ; 128: 343-350$

14 Burt AD, Portmann BC, Ferrell LD: MacSween's Pathology of the Liver, ed 5. Edinburgh, Churchill Livingstone Elsevier, 2007, p 130. 